\title{
HIGH-FREQUENCY RCS OF PERFECTLY CONDUCTING \\ OR COATED COMPLEX OBJECTS IN REAL TIME
}

\author{
Juan M. Rius*, M. Vall-1lossera*, Angel Cardama*
}

\begin{abstract}
This paper presents a new and original approach to compute high-frequency radar cross section (RCS) of complex radar targets in real time using a 3-D graphic workstation. The aircraft is modelled with I-DEAS solid modeling software using a parametric surface approach. High-frequency RCS is obtained through Physical Optics (PO), Method of Equivalent Currents (MEC), Physical Theory of Diffraction (PTD) and Impedance Boundary Condition (IBC). Multiple scattering between target surfaces is also considered.
\end{abstract}

\section{INTRODUCTION}

Numerical computation of RCS of large and complex radar targets is based on high-frequency approximations: the target is usually modelled in terms of facets and wedges, so that physical optics and physical theory of diffraction can be respectively applied to each facet and wedge Youssef(1) Domingo et al.(2). These classical techniques require very long CPU run time on powerful computers.

The objective of this paper is to show that real-time RCS computation is possible with a high-performance graphic workstation Rius(4): PO, PTD and IBC approximations are computed using the hardware capabilities of a graphics accelerator. Real-time computation is achieved through graphical processing of an image of the target present at the workstation screen. First-order reflections are obtained by rendering of the target with a local illumination algorithm, and multiple scattering with a global illumination one.

\section{TARGET MODELING}

A computer aided design package for geometric modeling of solids I-DEAS(3) has been used for modeling target geometry. The target is described as a collection of parametric surfaces, defined with two-dimensional NURBS (non-uniform rational B-splines).

Classical RCS analysis packages that describe the target in terms of facets and wedges (1) (2) have a potential limitation when a large number of facets might be required, which may exceed declared array sizes or which can lead to longer CPU run times. On the other hand, parametric surface modeling of the target impose weaker storage memory requirements that the faceting approach, and the modelled surface adjusts more accurately to the real target surface.

\section{HARDWARE GRAPHICAL PROCESSING IN REAL TIME}

The following high-frequency scattering phenomena are considered by the real-time graphical processing approach (4):

- Reflection at perfectly conducting surfaces by physical optics approximation.

- Reflection at coated surfaces by physical optics and IBC approximations.

- Diffraction at edges by method of equivalent currents using PTD diffraction coefficients.

- Multiple reflections between surfaces by a global illumination method.

Figure 1 shows the relationship between the different graphical processing algorithms and high-frequency approximations.

* Dep. Teoría de la Señal y Comunicaciones, E.T.S.I.Telecomunicación, U.P.C.

Apdo. 30002, 08080 Barcelona, Spain 


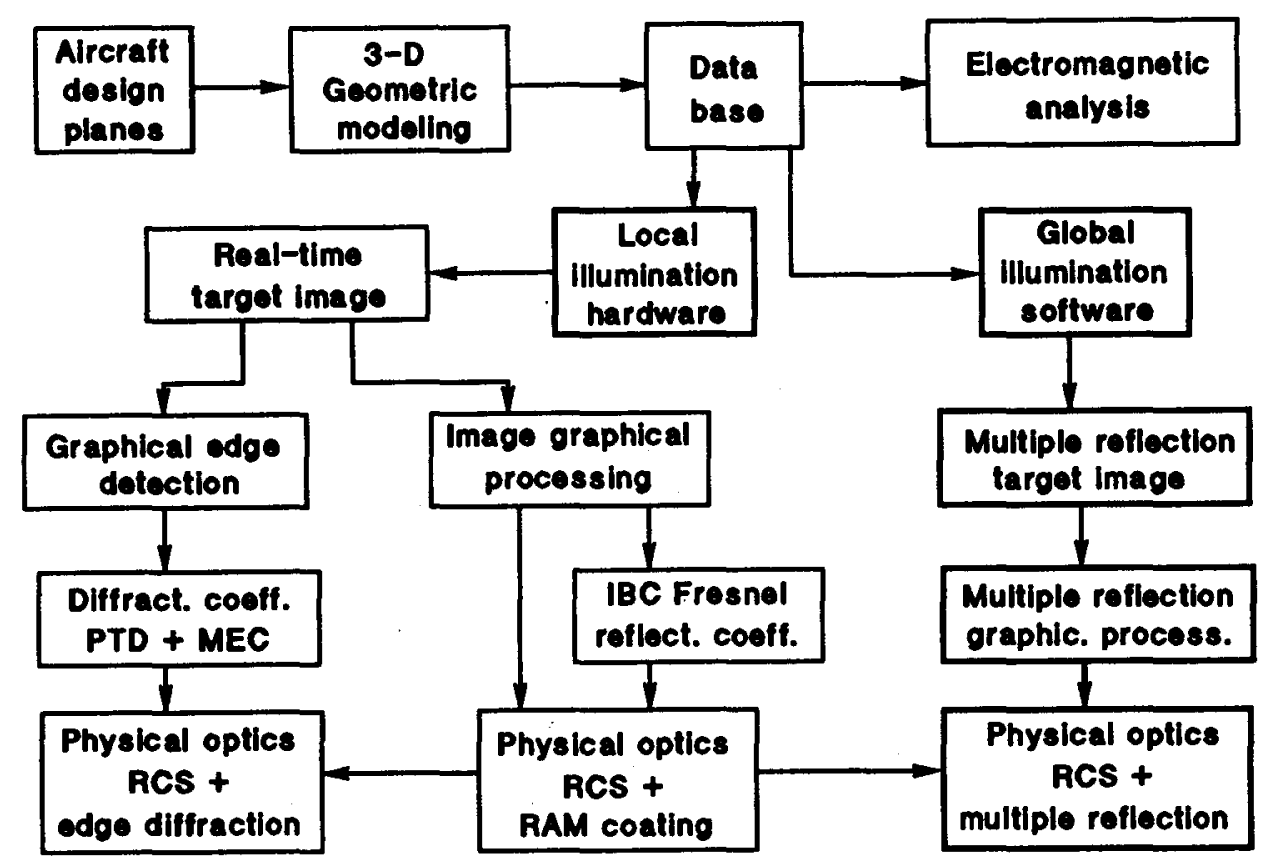

Figure 1: Real-time graphical processing algorithms and high-frequency approximations

\section{Physical optics}

The main difficulty to compute the physical optics surface integral by classical techniques -faceting approach- (1) (2) is the detection of shadow regions. This difficulty can be easily overcome using the hidden surface removal capabilities of a graphic workstation, which can be performed by hardware if a graphics accelerator is present. Hidden surfaces are removed comparing the distance from the observer to each pixel to be drawn with the distance of the previously drawn pixel in the same display location, stored in a portion of RAM called "z-buffer".

A photorealistic drawing of the target is made from a view-point coincident with radar position, so that shadowed regions are not displayed. A directional light source is defined on the same direction as the incident wave-front. If the target surface is modelled to have only diffuse reflection of light, it results that the brightness of each pixel on the drawing is proportional to its PO contribution to the total RCS (4).

Therefore, the physical optics surface integral can be evaluated as the coherent addition of the brightness of all the pixels on the display. The phase of each pixel contribution can be easily obtained from the distance to the observer stored in the "z-buffer".

\section{Method of equivalent currents}

In order to compute high-frequency edge diffraction by method of equivalent (MEC) currents, the following graphical processing algorithm has been developed (4):

1- An image of the target is made at the workstation screen. Hidden edges are removed by the graphics accelerator hardware, so that only visible ones are displayed.

2- The surface unit normal at each pixel of the image is computed in the following way: Three light sources (Red, Green and Blue) are defined with incidence from $x, y, z$ axis directions. If surface reflection is diffuse, the brightness of each colour $(R, G, B)$ is respectively equal to the $x, y$ and $z$ component of the unit normal to the surface.

3- Edges are detected on the target image as discontinuities of the unit normal to the surface.

4- For each pixel on detected edges, interior wedge angle and incidence direction relative to wedge faces are computed from the unit normal to each face of the wedge. Monostatic PTD diffraction coefficients are then obtained using a very simple linear approximation, which saves run-time avoiding trigonometrical functions (4). 
5- Line integral of MEC is evaluated using the PTD coefficients for each pixel and the distance to the observer stored in "z-buffer".

\section{Impedance boundary condition}

Radar absorbent coatings are considered through impedance boundary condition (IBC) and physical optics approximations, which lead to a very simple formulation of PO surface integral with Fresnel reflection coefficients. Reflection coefficients are formulated a function of equivalent surface impedance, which is obtained through a transmission line equivalent circuit.

In order to compute the reflection coefficients, the angle between incidence direction and unit normal surface is obtained by graphical techniques in the same way as in MEC-PTD graphical processing algorithm (4).

\section{Multiple scattering}

Multiple scattering contribution to RCS has been computed by global illumination radiosity method Goral et al.(5), which models the interaction of light between perfectly diffuse surfaces, with a reduced computational effort. The well-known technique of ray-tracing has not been used because of its prohibitive computational cost.

Due to the incoherent nature of the radiosity method, based on an energy equilibrium basis, RCS with multiple scattering must be obtained as the incoherent addition of first order physical optics contribution plus multiple scattering radiosity contribution. The later is computed by incoherent summation of the increment of brightness of each pixel due to global illumination rendering of the target (4).

\section{Performance}

Graphical processing has the following advantages over classical techniques (1) (2):

- Hardware graphics accelerator removes hidden surfaces and edges so that they do not contribute to surface or line integrals.

- Evaluation of surface and line integrals (PO and MEC) independent of target complexity.

- CPU time and RAM requirements independent of target electrical size and complexity.

- Real-time computation if hardware graphics accelerator is used.

- Target can be modelled by parametric NURB surfaces, requiring less mass storage memory that the faceting approach, and adjusting more accurately to the real target surface.

On a HP-380 Turbo SRX high performance graphic workstation with hardware graphics accelerator, the CPU run time is about 10 microseconds/pixel/angle for first order PO and 30 microseconds/pixel/angle for PO+PTD for both polarizations, while RECOTA package (1), developed by Boeing Aerospace, takes about 22 milliseconds/facet/angle on a VAX 11/785, including multiple scattering and second-order effects.

The results obtained by radiosity graphical processing require a CPU time of about 5 to 10 times greater than first order physical optics graphical processing. Although this is not a real time algorithm, it is still much faster than classical multiple scattering methods (1) (2), so we can consider it a quasi-real time algorithm.

\section{RESULTS}

In this section, real-time graphical processing results for electrically large and complex radar targets will be compared with those obtained by classical methods (1) (2) and some measurements performed by Boeing Aerospace (1). Further graphical processing results for simple objects with RAM coatings have been published, for example, in JINA' 90 Workshop (6).

In fig. 1 the graphical processing prediction for a Boeing 727 aircraft is compared with RCS measurements (Boeing Airplane Company). The agreement is very good. The graphical processing RCS has been obtained in real time (2 seconds/angle), while classical techniques (1) (2) require several hours due to the large size of the target $(128 \lambda)$. 
Fig. 2 shows the results for NACA 3317 airfoil section. Graphical processing is compared with RECOTA predictions (1). The agreement is excellent.

Finally, fig. 3 shows the RCS of a generic missile model $40 \lambda$ long: a) first-order PO graphical processing results compared with TOTAL code prediction (2), b) RECOTA prediction (1) and c) radiosity graphical processing results compared with first order PO and experimental measures (1).

In fig. $3 \mathrm{a}$ ) and $\mathrm{b}$ ) the agreement between graphical processing first order PO, TOTAL (2) and RECOTA (1) results is very good.

In fig. $3 \mathrm{c}$ ) note that first order RCS is lightly smaller than the measured one at angles between $50^{\circ}$ and $70^{\circ}$, in which a double reflexion occurs between the fuselage and the leading edge of the wing. Note also that due to the incoherent nature of radiosity method, it can not predict phase cancellations on reflected fields, so that RCS nulls disappear.

\section{Conclusions}

- First order PO approximation predicts with reasonable accuracy RCS of real radar targets.

- Real-time results are possible with hardware graphical processing.

- MEC with PTD coefficients improves PO results for both polarizations when edge diffraction is dominant.

- Radar absorbent materials analysis through IBC is valid only for surface reflection, when a specular reflection point exists. Impedance wedges should be treated by a higher-order approximate boundary condition.

- Although multiple reflection effects are of secondary importance when RCS of real targets is computed, they can be included if a global illumination method is used for graphical processing. Due to the incoherent nature of radiosity algorithm, it can not predict phase cancellations on reflected fields, so that RCS prediction is always in excess of the measured one.

- Real-time RCS computing software can be integrated with CAD geometric modeling package (3), thus providing an efficient tool for interactive modeling, design and analysis of aircraft with RCS specifications.

\section{ACKNOWLEDGEMENTS}

This work has been supported by the Spanish "Comisión Interministerial de Ciencia y Tecnologia" (CICYT) under the proyect: ACCION ESPECIAL PRONTIC "Programa de investigación aplicada para el desarrollo y validación de métodos de cálculo numérico para la predicción y análisis de las características de los ecos radar (RCS) y su reducción", TIC 88-288E. Management: "Dirección General de Telecomunicaciones" (D.G.Tel). Coordination: C.A.S.A.

\section{REEERENCES}

(1) N.Youssef, "Radar Cross Section of Complex Targets", 1989, IEEE Proc., Vol.77, No.5, pp.722-734

(2) M.Domingo, F.Rivas, M.F.Cátedra, R.Abad, J.I.Casado, P.L.Primo del Val, R.Torres, "Programa base de GTD-PTD para el cálculo de la RCS de cuerpos conductores modelados por parches planos y teniendo en cuenta simple y doble reflexión, difracción en aristas y eliminación de zonas ocultas", 1990, Procc. V Symp. Nacional Comité Español URSI, pág. 185-189

(3) "I-DEAS Geomod Users Guide", Structural Dynamics Research Corporation (SDRC), Milford, OH 45150

(4) J.M.Rius, "Sección recta de blancos radar complejos en tiempo real", 1991, Tesis Doctoral, Universitat Politecnica de Catalunya

(5) C.M. Goral, K.E. Torrance, D.P. Greenberg, B. Battaile, "Modeling the Interaction of Light Between Diffuse Surfaces", 1984, ACM Computer Graphics, Proceedings of SIGGRAPH

(6) "RCS of perfectly conducting or coated bodies", Workshop JINA'90, Nice, 16th November 1990 


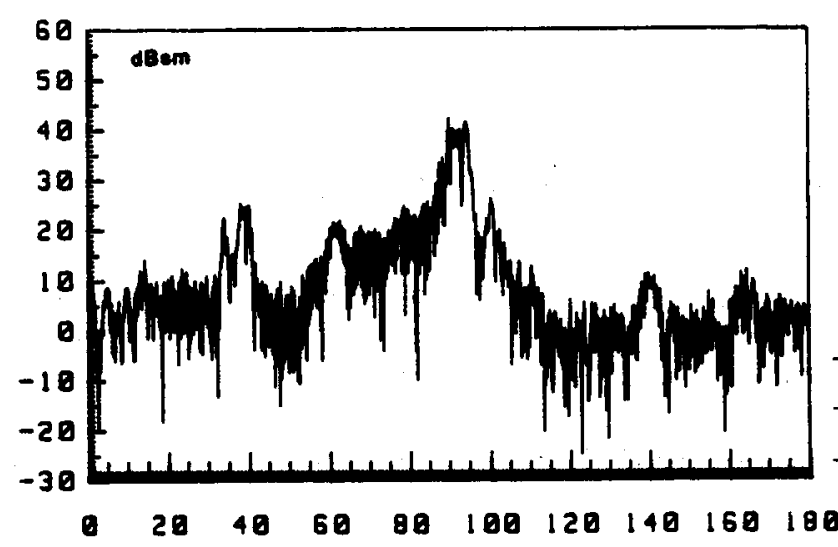

a) Physical optics graphical processing

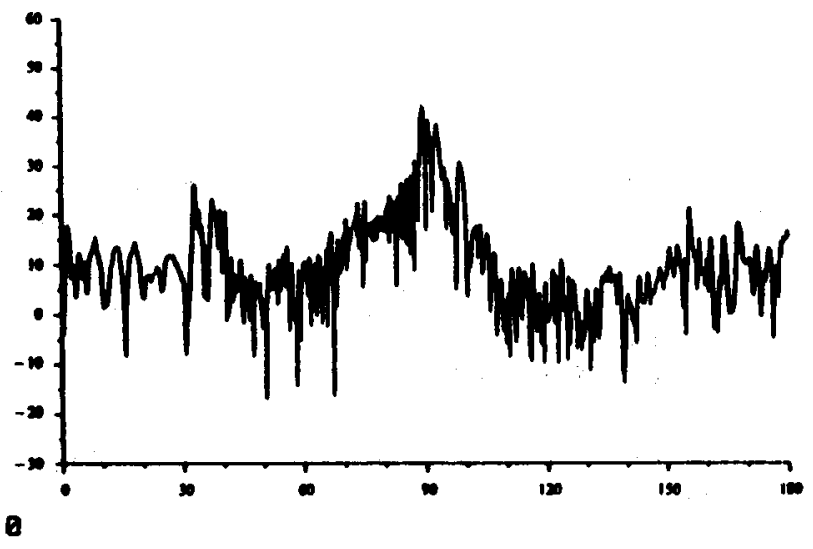

b) Measured RCS for horizontal polarization

Fig. 1: RCS of Boeing 727-100C at $0.94 \mathrm{GHz}$ :
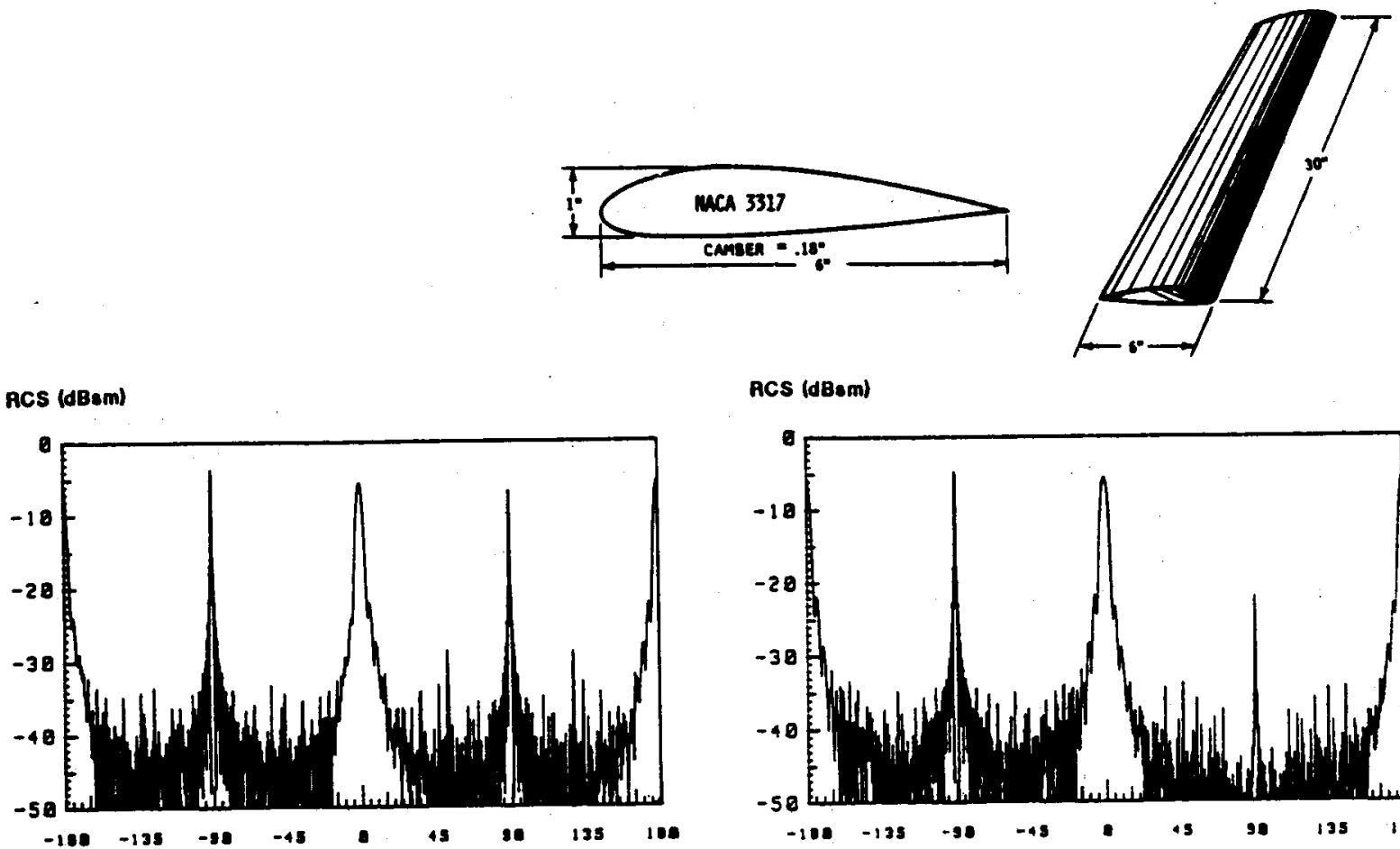

RCs (dBam)

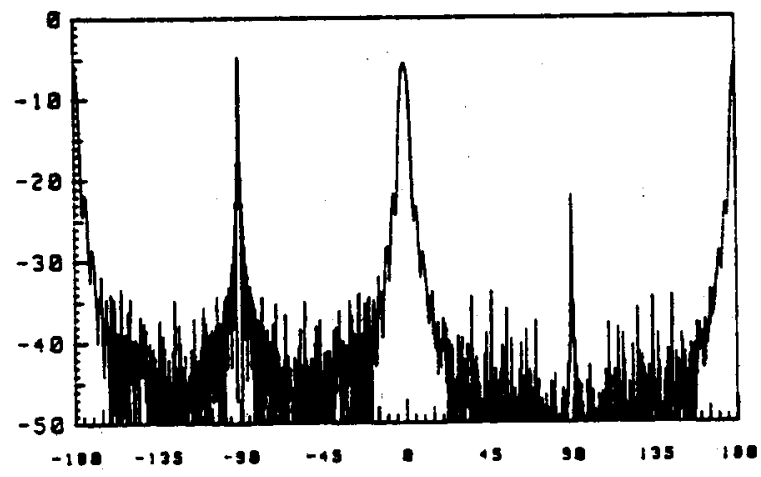

a) Horizontal polarization PO + PTD graphical processing

b) Vertical polarization

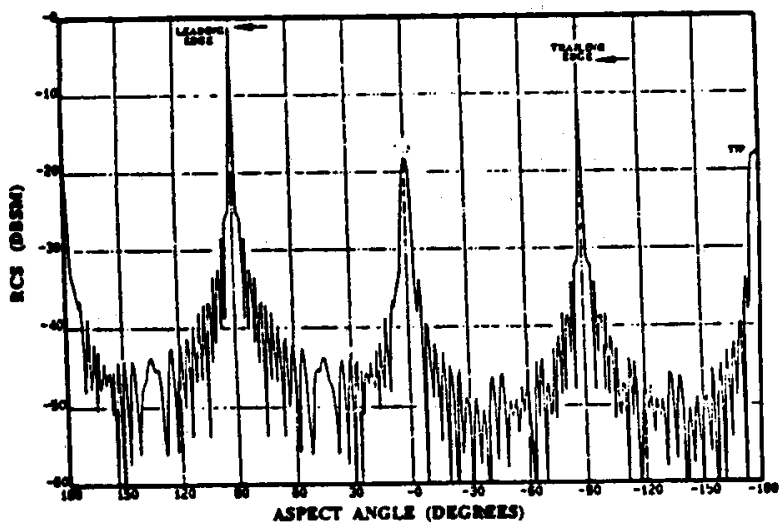

c) Horizontal polarization

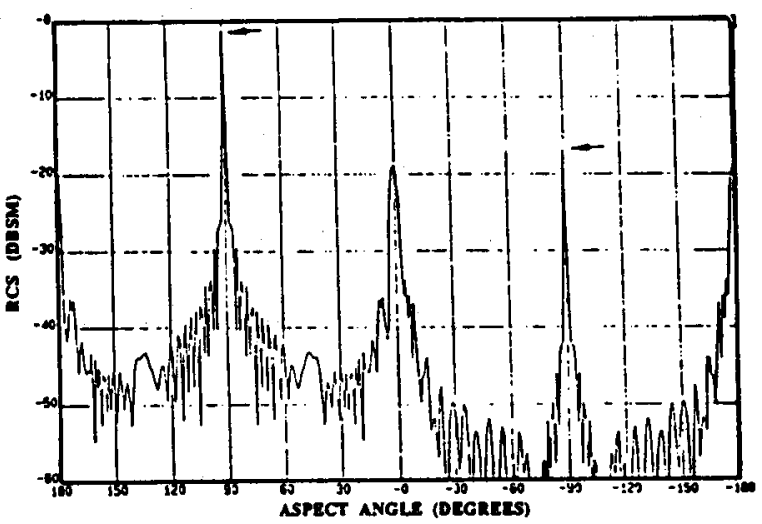

d) Vertical polarization

Fig. 2: RCS of NACA 3317 airfoil. 


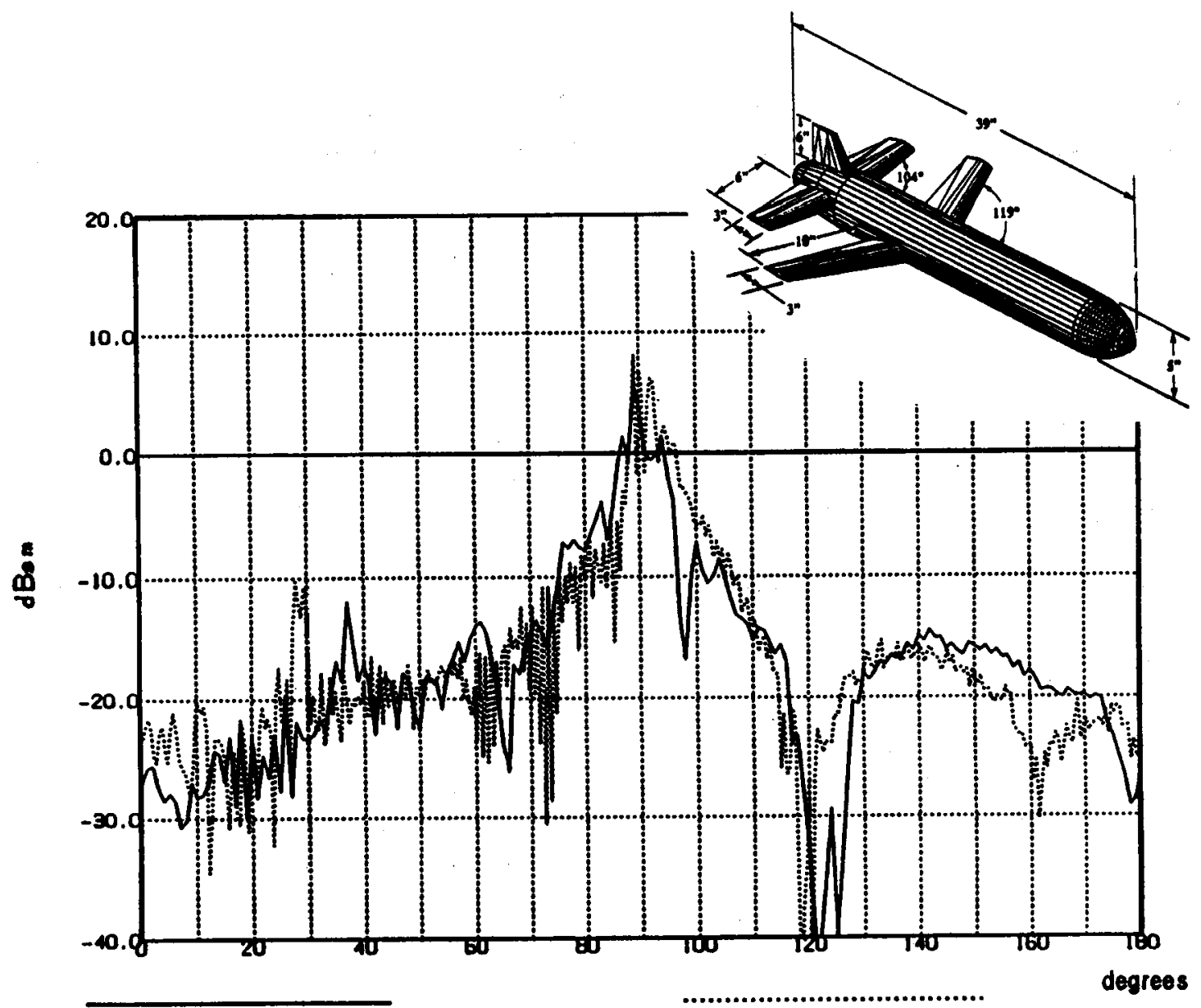

TOTAL : P.O. + PTD + Multiple Scatering Verlical Polarization

Real Time P.0. Graphical Processing

a) first-order PO graphical processing results compared with TOTAL code prediction (2)

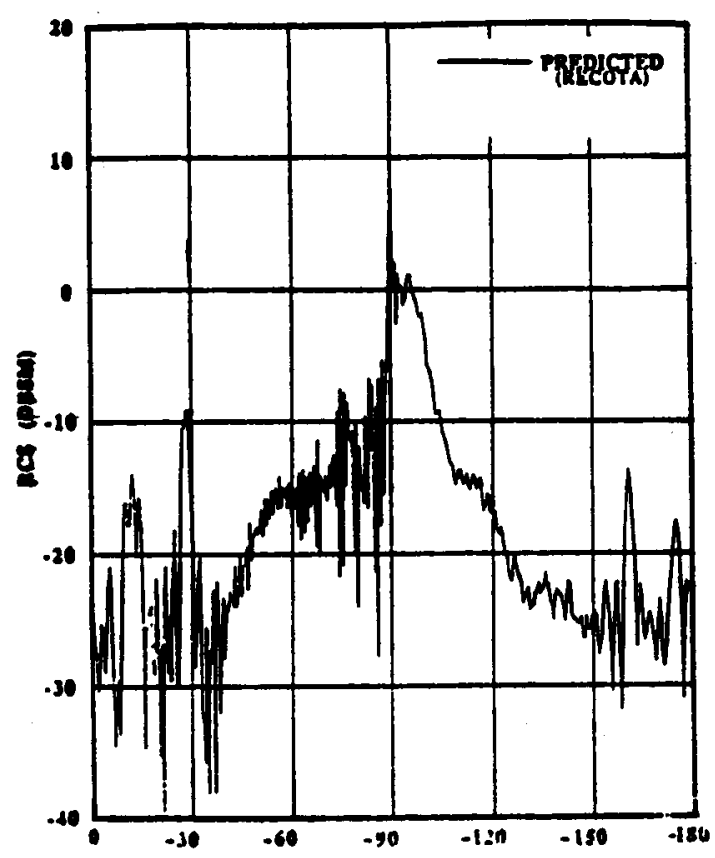

b) RECOTA prediction (1)

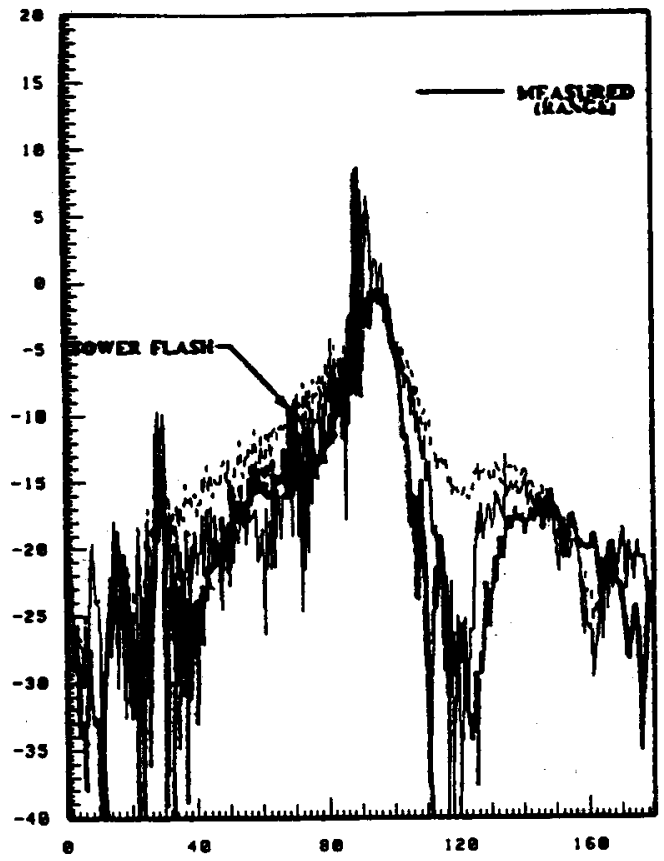

c) Dot line: Radiosity graphical processing Thin line: Physical optics graphical processing Thick line: Experimental measurement (1)

Fig. 3: RCS of missile of $1 \mathrm{~m}$. length at $12 \mathrm{GHz}$ 